\title{
Unit Commitment Based on Risk Assessment to Systems with variable Power Sources
}

\author{
P. M. Fonte \\ Electrical Engineering Department \\ Lisbon Superior Engineering Institute, ISEL \\ Lisbon, Portugal \\ pfonte@deea.isel.pt
}

\author{
Cláudio Monteiro ${ }^{1}$ \\ Fernando Maciel Barbosa ${ }^{1,2}$ \\ ${ }^{1}$ FEUP - Engineering Faculty, University of Porto \\ 2 INESC TEC \\ Porto, Portugal \\ cdm@fe.up.pt,fmb@fe.up.pt
}

\begin{abstract}
This paper presents the development of a complete methodology for power systems scheduling with highly variable sources based on a risk assessment model. The methodology is tested in a real case study, namely an island with high penetration of renewable energy production. The uncertainty of renewable power production forecasts and load demand are defined by the probability distribution function, which can be a good alternative to the scenarios approach. The production mix chosen for each hour results from the costs associated to the operation risks, such as load shed and renewable production curtailment. The results to a seven days case study allow concluding about the difficulty to achieve a complete robust solution.
\end{abstract}

Index Terms-Power generation scheduling, risk assessment, uncertainty.

\section{INTRODUCTION}

Increasing introduction of electric energy production with Renewable Energy Sources (RES), and mostly those with high variability, has created several challenges to the energy networks operators, especially in the scheduling. This problem is boosted in low power networks, particularly in islands without any connection to continental networks. Large variations on renewable production can introduce stability problems in the network, which can originate generation or load shed and, at limit, black-outs a strong possibility[1]. When available, the RES production allows thermal production decrease, especially during the peak load periods. Optimizing the number and the power of the on-line thermal units lowers cost and emissions. On the other hand, an extreme reduction of the thermal committed capacity can lead to a situation where the spinning reserves are not sufficient to handle with great variations of load, renewable production or generation outages. Therefore, due to the uncertainty in load and renewable production forecast, it is sometimes hard to find a completely robust/economic scheduling solution. With this into consideration and for security, scheduling is generally done by a conservative way, with low risk, although sometimes far away from an optimal operation. As such there is the necessity to introduce uncertainty of load/RES in scheduling for achieving a better management of the thermal unit's commitment. The stochastic programming is an approach widely used to deal with the generation scheduling under uncertainty applying recourse problems, chance-constrained or robust optimization, with uncertainty described by scenarios [2][17]. The scenario-based approach demands a great number of realizations in order to capture the temporal interdependence of the probabilistic behavior of the uncertainty. One of the main problems of this approach is that it is time consuming to solve all scenarios, being necessary to appeal to some kind of scenario reduction. To overcome this problem, this work develops a short-term scheduling approach to be used in insular power grids based on risk assessment, addressing the increase of variability and uncertainty created by RES.

\section{DESCRIPTION OF THE METHODOLOGY}

The proposed generation scheduling is designed to minimize the sum of the estimated costs based on risk cost analysis. These costs result from the estimated normal operation cost plus the estimated cost of operating outside normal conditions. It is understood as "abnormal" conditions if there is the necessity of load shed due to the lack of available thermal production or RES curtailment caused by the lack of load. The risk of load shed or RES curtailment and thermal production below the technical minimums are used to define the objective function, as well as the probability of the thermal generators operating inside the appropriated ranges.

Contrary to widely used scenarios-based approach, in this work it is proposed the probabilistic estimation of costs based on estimation risk, directly using the probability density function of the random variables. Knowing the probability function of net load $\left(L_{N}\right)$, obtained by load minus the renewable production $(L-R E S)$ [1],[5],[6],[9],[15], for each hour $h$ of the scheduling period, the ability of each thermal GENeration mix SET (GENSET) to meet the net load is verified. Notice that in the risk assessment approach there are no infeasible solutions, only more or less costly solutions. These decisions have to be made to accept a risk as long as it 
can be technically and financially justified, thus all the combinations have to be tested.

\section{A. Equivalent optimal generation unit}

The thermal power capacity installed in the island under study is composed by 8 thermal units divided in 2 groups of 4 units with the same rated power. In a restricted analysis of available power, the 256 possible combinations can be reduced to 24 regardless of which thermal units are on-line. This allows creating a much more tractable dataset (the hypothesis of all units are off-line was not considered). The cost function of thermal units were defined as continuous and convex second order functions[19] which allowed to create a dataset with all possible combinations of thermal mixes, defining a GENSET to each combination with the respective limits of generation. The unique restrictions taken into account in this formulation were the minimum and maximum production limits of each unit and the obligation of the total production to be equal to the load. For sake of simplicity the problem is formulated and solved without considering transmission losses. To each GENSET is solved an economic dispatch (ED) for different values of net load which allows to define an equivalent generator. The added value of this approach is the easiness and the calculation velocity of power production that each unit must generate. At the same time, the fuel consumption cost is also calculated. Thus, it is not necessary to run any ED during the on-line scheduling which will reduce the computation time of the process. Although exhaustive and very time consuming, this procedure is done only once, being updated when there is a change in the number or rated power of thermal units.

\section{UNIT COMMITMENT BASED ON RISK ASSESSMENT}

For each hour ahead, knowing the $p d f$ of the net load for each hour ahead and all combinations of GENSETs, is done the risk assessment for each GENSET and the expected risk costs are computed. After, is done a $(N-1)$ contingency analysis regarding the outage of a single thermal unit. It is assumed that the failure of a unit cannot be repaired or replaced within this period. This process is done in an independent way for each hour ahead. Knowing all the risk costs associated to each GENSET to each hour ahead $h$, the start-up costs are integrated using a forward dynamic programming, resulting in the solution with lower cost with risk embedded. In the methodology proposed in this work it is also not considered a predefined value for the reserves, since reliability and operational risk minimization are expected to lead to solutions with enough levels of dynamic reserves.

During the evaluation of each GENSET, depending of the net load $p d f$, there are different operation risk areas. In Fig. 1 a generic net load $p d f$, as well as, the minimum $\left(\min _{\text {GENSET }}\right)$ and maximum $\left(\max _{\mathrm{GENSET}}\right)$ limits of a generic GENSET are shown.

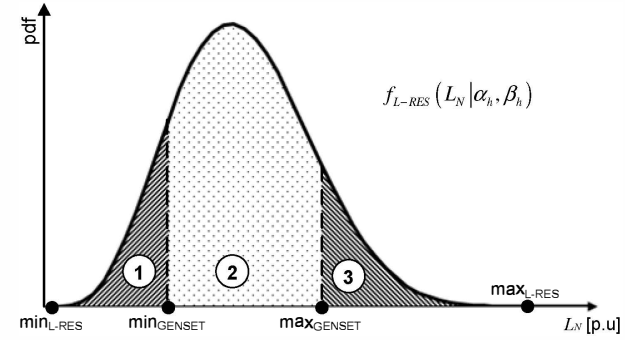

Figure 1. Uncertainty associated to a specific committed GENSET

\section{A. Probability of operation below the minimum GENSET limit}

Area 1 represents the risk of thermal units to operate under its technical minimum $\left(\min _{L-R E S}<\min _{\text {GENSET }}\right)$ and represents the risk of RES curtailment. The probability of RES curtailment $\operatorname{prob}(W C)_{h}$ in hour $h$ is calculated by (1), where $F_{L-R E S, h}$ represents the $c d f$ of net load for hour $h$.

$\operatorname{prob}(W C)_{h}=\left\{\begin{array}{cc}0, & \min _{\text {GENSET }} \leq \min _{L-R E S, h} \\ F_{L-R E S, h}\left(\min _{\text {GENSET }}\right) & \min _{L-R E S, h}<\min _{\text {GENSET }}<\max _{L-R E S, h} \\ 1, & \min _{G E N S E T} \geq \max _{L-R E S, h}\end{array}\right.$

(1)

\section{B. Probability of operation above the maximum}

\section{GENSET limit}

On the other hand, area 3 corresponds to the probability of the net load being greater than $\max _{\text {GENSET }}$ (total thermal capacity of the mix). Therefore, there is the probability of some load not be supplied in hour $h$ (load shed), calculated by (2).

$$
\operatorname{prob}(L S)_{h}=\left\{\begin{array}{cc}
1, & \max _{\text {GENSET }} \leq \min _{L-\text { RES }, h} \\
1-F_{L-R E S, h}\left(\max _{G \text { GNSERT }}\right) & \min _{L-R E S, h}<\max _{\text {GENSET }} \leq \max _{L-\text { RES, }, h} \\
0, & \max _{\text {GENSET }} \geq \max _{L-\text { RES }, h}
\end{array}\right.
$$

\section{Probability of operation inside GENSET limits}

The area bounded by the RES curtailment probability and load shed probability is the area of GENSET's normal operation (NO) and it is defined by GENSET technical limits $\left(\min _{\text {GENSET }}\right.$ and $\max _{\text {GENSET }}$ ), calculated by (3).

$$
\operatorname{prob}(N O)_{h}=1-\left[\operatorname{prob}(W S)_{h}+\operatorname{prob}(L S)_{h}\right]
$$

\section{RES curtailment}

In this power system, the thermal units have the role of maintain the frequency, and even if the RES production is enough to feed the load, there is always a minimum value of thermal production that must be kept on-line. In the case of high levels of power production by RES and low load can lead to the necessity of RES curtailment to increase the net load to values above the GENSET's minimum generation limits. In the case study presented in this paper when there is the risk of thermal units to work below their minimums, the wind curtailment is an option to take in consideration. Even 
so, it is necessary to determine if there still is the risk of thermal units to operate under the technical limits after total wind curtailment. Considering load and wind generation independent random variables, another indicator is introduced, $L_{N W}=L-(G E O+H)$, which represents the resulting net load after the partial or total wind curtailment. The indices $G E O$ and $H$ will by explained ahead. The new variable $L_{N W}$ will originate another Beta $p d f$ defined by the parameters $\alpha_{h 1}$ and $\beta_{h 1}$, limited by the limits $\min _{L-(H+G E O)}$ and $\max _{L-}$ $(H+G E O)$. With wind curtailment, the amount of RES is reduced and, consequently, the need of thermal production is increased. In fig. 2 the $p d f$ of net load with and without wind curtailment are shown. This situation reduces, but does not avoid, the risk of thermal units continue operate below their minimums. Depending on the available wind power production for the hour $h$, the curtailment capacity should be different and, even with total wind curtailment, it could not be enough to raise the minimum value of the net load to a value higher than $\min _{\text {GENSET }}$. Thus, it is necessary to calculate the new risk, by calculating the new probability $\operatorname{prob}(\min \mid W C)_{h}$. This is based on (1) defining the new Beta distribution with parameters $\alpha_{h 1}$ and $\beta_{h 1}$.

After the definition of the different risk areas for all GENSET, the power values must be computed in order to calculate the cost assigned to each area and calculate the total cost associated to each GENSET at hour $h$.

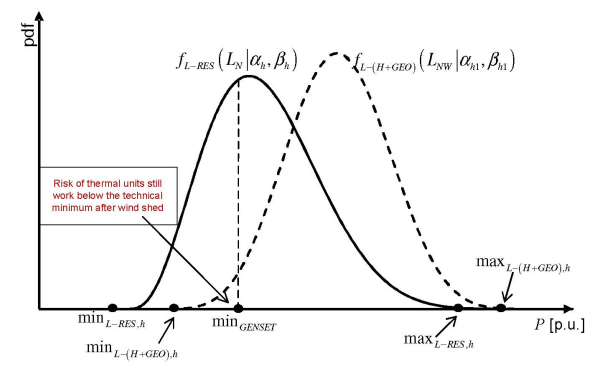

Fig. 2. $P d f$ of $L_{N}$ with $[L-(H+G E O)]$ and without wind curtailment (L-RES).

In this situation there are two hypotheses: the quantity of available wind production is higher than the curtailment necessity (being necessary to curtail only a percentage of available power), or the wind power capacity to curtail is lower than the needed.

To estimate $P(W C)_{h}$, it is necessary to obtain the inverse of the Beta $c d f$ of the $L_{N}$ as shown in figure 3 , and the wind power forecast.

$$
P(W C)_{h}=\min _{G E N S E T}-\min \left[F_{L-R E S, h}^{-1}\left(\frac{\operatorname{prob}(W C)_{h}}{2}\right), \hat{P}_{w}\right]
$$

With this inverse function it is possible, assigning some probability, to know the power to curtail. The result of (4) will be the minimum of these values because the wind power available to curtail is limited by the wind power forecast.

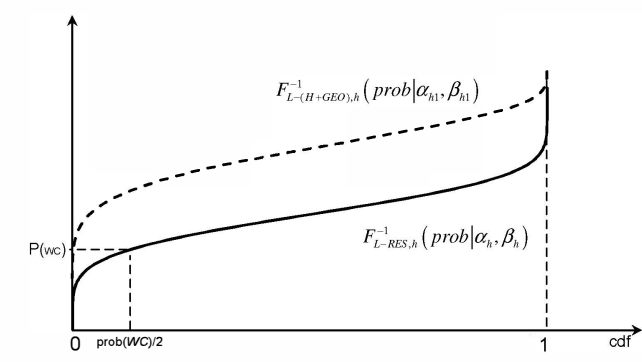

Figure 3. Inverse $c d f$ of $L_{N}$ with (L-(H+GEO)) and without wind curtailment L-RES

If the total wind curtailment is not enough to guarantee that the thermal units are not operating below the minimum, it is possible to estimate the value that the thermal units are producing below the minimum by (5). If the $\min _{\text {GENSET }}$ is lower or equal to $\min _{L-(H+G E O)}$ the result is zero. If the $\min _{\text {GENSET }}$ is higher or equal $\max _{L-(H+G E O)}$ the result is calculated by the third term of (5) else the value results from the second term. On the opposite case, if there is a risk of load shed $(\operatorname{prob}(L S)>0)$, the value $P(L S)_{h}$, is calculated by (6).

$$
\begin{aligned}
& P(\Delta \operatorname{prob} \mid W C)_{h}=\left\{\begin{array}{l}
0 \\
\min _{G E N S E T}-\left\{F_{L-(H+G E \bullet), h}^{-1}\left[\frac{\operatorname{prob}(\min \mid W C)}{2}\right]\right\}(5) \\
\min _{G E N S E T}-F_{L-(H+G E O), h}^{-1}(0,5)
\end{array}\right. \\
& P(L S)_{h}=F_{L-R E S}^{-1}\left(1-\operatorname{prob}(L S)_{h}+\frac{\operatorname{prob}(L S)_{h}}{2}\right)-\max _{G E N S E T}
\end{aligned}
$$

Finally, the calculation of power produced under normal operation, when $0<\operatorname{prob}(W C)_{h}<1$ and $0<\operatorname{prob}(L S)_{h}<1$, is done by (7)

$$
P(N O)_{h}=F_{L-R E S}^{-1}\left[\frac{1-\operatorname{prob}(L S)_{h}+\operatorname{prob}(W C)_{h}}{2}\right]
$$

\section{A. Risk cost analysis (without contingencies)}

To conclude the evaluation of performances of each GENSET (so far without contingencies), the risk costs related with each risk assessment done so far are computed. The risk cost of wind curtailment is calculated by (8) whereas the risk cost of load shed is done by (9),

$$
\begin{gathered}
C_{W C, h}=\operatorname{prob}(W C)_{h} \times P(W C)_{h} \times C_{W C} \\
C_{L S, h}=\operatorname{prob}(L S)_{h} \times P(L S)_{h} \times C_{L S}
\end{gathered}
$$

where, $C_{W C}$ and $C_{L S}$ are, respectively, the wind curtailment and load shed cost. This costs, in $€ / \mathrm{MWh}$, are constants independently of the amount curtailed. When, after the total wind curtailment, there still is violation of the minimum limits of a GENSET, the risk cost is calculated by (10), where the cost $C_{M I N_{-} G E N}$ is considered constant (there is 
not enough information concerning to the consumption below the minimum power).

$$
C_{\text {min| } \mid W C, h}=\operatorname{prob}(\min \mid W C)_{h} \times P(\Delta p r o b \mid W C)_{h} \times C_{M I N_{-} G E N}
$$

The thermal generators' risk cost of normal operation are calculated by (11), where $F[$.$] is the equivalent optimal$ generation unit fuel consumption function. $C_{F U E L}$ is the fuel cost.

$$
C_{\text {Ne }, h}=\left\{\begin{array}{l}
\operatorname{prob}(N O)_{h} \times F\left[P(N O)_{h}\right]+ \\
\operatorname{prob}(L S)_{h} \times F\left(\max _{\text {GENSET }}\right)+ \\
\operatorname{prob}(W C)_{h} \times F\left(\min _{\text {GENSET }}\right)+ \\
\operatorname{prob}(\min \mid W C)_{h} \times F\left(\min _{\text {GENSET }}\right)
\end{array}\right\} \times C_{F U E L}
$$

At the end, the risk cost for a given GENSET at hour $h$ based on risk assessment is calculated by (12).

$$
C_{\text {GENSET }}=C_{W C, h}+C_{L S, h}+C_{\min \mid W C, h}+C_{N O, h}
$$

There is an important issue that must be highlighted; the results from (12) are not the real costs. They are only risk costs calculated in order to define the scheduling. The final real costs must be calculated with measured values.

\section{E. Contingency analysis}

The contingency analysis was limited to one unexpected thermal unit outage $(N-1)$. In this study there are only two types of thermal units. Those with lower rated power were defined as $G_{S}$ and those with higher rated power were defined as type $G_{B}$. Following this approach, the evaluation of a $N-1$ contingency is done by(13). The parameter $n_{S}$ and $n_{B}$ represents the number of on-line units of type $G_{S}$ and $G_{B}$, respectively, while indices $(n-1) S, h$ and $(n-1) B, h$ represents the outage of a unit of each type, at hour $h$, of the GENSET under evaluation. The parameter $n_{U N I T}$ represents the total units of the GENSET under evaluation, and $U$ the probability of contingency.

$$
\begin{aligned}
& C_{\text {GENSET }(N-1), h}=(1-U)^{n_{U N I T}} \times C_{G_{\text {GENSET }} n S_{-} n B, h}+(1-U)^{n_{U N I T}} \times \\
& {\left[n_{S} \times U \times C_{G E N S E T_{-}(n-1) S_{-} n B, h}+n_{B} \times U \times C_{G E N S E T_{-} n S_{-}(n-1) B, h}\right]}
\end{aligned}
$$

With this technique, and with the database created offline, is possible to calculate the contingency cost, since the equivalent cost functions of each GENSET are already known. For instance, if the GENSET_3 $3 \mathrm{G}_{\mathrm{S}_{-}} 3 \mathrm{G}_{\mathrm{B}}$, lose a unit type $G_{S}$, the new cost can be calculated searching in the data base the cost related with the GENSET_2 $\mathrm{G}_{\mathrm{S} \_} 3 \mathrm{G}_{\mathrm{B}}$. Previous evaluations have two exceptions, which happen when there only is one on-line unit, as GENSET_0G $\mathrm{G}_{\mathrm{S}} 1 \mathrm{G}_{\mathrm{B}}$ or GENSET_ $1 \mathrm{G}_{\mathrm{S}_{-}} 0 \mathrm{G}_{\mathrm{B}}$. It is considered that the loss of all thermal generation will conduce to a blackout. In this case, it is considered a constant blackout cost $\left(\mathrm{C}_{\mathrm{BO}}\right)$ plus the cost of net load shed, as in (14).

$$
C_{G E N S E T(N-1), h}=(1-U) \times C_{G E N S E T, h}+U\left[\left(L_{N}\right) \times C_{L S, h}+C_{B \bullet}\right]
$$

After the knowledge of all the risk costs to each hour $h$ of the scheduling period, a forward dynamic programming is done. As there is no infeasible solution, all the GENSETs should be tested which should increase the dimensionality of the problem. To avoid this situation a heuristic method is proposed. The solutions of each period $h$ are ordered from the lowest to the highest cost. Departing from the lowest, only the solutions which costs are inside of a threshold are tested. This way, only solutions near the best solution at each state are tested, with "paths" not fulfilling the entire scheduling being eliminated. However, as all heuristics, there is no certainty that the most economical solution is achieved; the compensation is the velocity of the process. In fig. 4 the complete flowchart of the multi-period unit commitment based on risk assessment is shown.

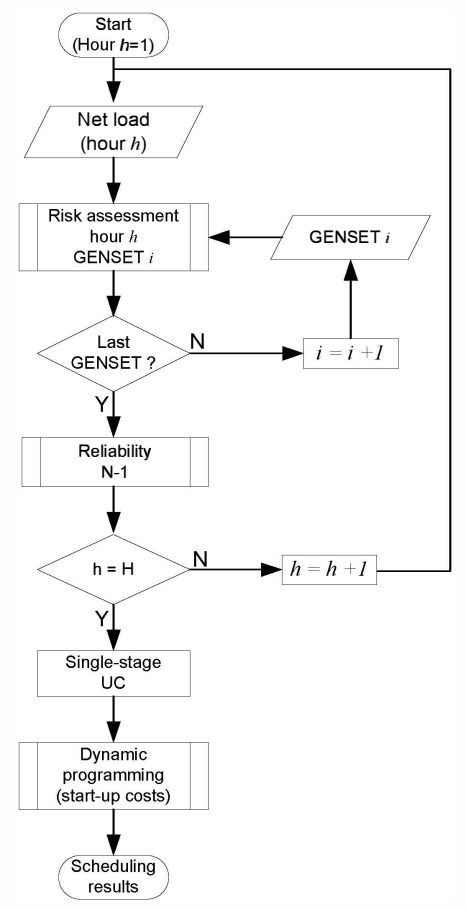

Fig. 4. Flowchart representing the proposed UC

\section{CASE STUDY}

The presented case study is performed under real conditions in the Portuguese Island of São Miguel. The production mix is composed by thermal, hydro, geothermal and wind power plants. In table I is depicted the power sources, the number of units and the total power of each source. The measured data related with power production and load demand was provided by the system operator, while the renewable power forecasts for wind, hydro and geothermal, as well as, load forecasts were provided by Smartwatt, Solutions for Energy Systems (www.singular.smartwatt.net). 
TABLE I

RATED POWER FOR EACH POWER SOURCE

\begin{tabular}{ccc}
\hline \hline Source & (\#units) & total power \\
\hline Fuel & 4 & $28 \mathrm{MW}$ \\
Wind & 4 & $64 \mathrm{MW}$ \\
Small hydro & 9 & $9,4 \mathrm{MW}$ \\
Geothermal & 7 & $5 \mathrm{MW}$ \\
\hline \hline
\end{tabular}

The unit commitment was solved for 24 hours periods and repeated for a 7 days period, between $0 \mathrm{~h} 00$ of February $25^{\text {th }}$ up to $23 \mathrm{~h} 00$ of March $3^{\text {rd }}, 2014$. With the choice of 7 days study it is intended to test the behavior of the proposed method for different days of the week. The power forecasts are received at $0 \mathrm{~h} 00$ of each day with a 30 hours horizon. In table II the production limits of thermal units and the parameters of fuel consumption functions are shown.

TABLE II

PARAMETERS OF THE THERMAL UNITS

\begin{tabular}{ccc}
\hline \hline \multirow{2}{*}{ Units } & Parameters & Values \\
& $\mathrm{P}_{\min }$ & $3848 \mathrm{~kW}$ \\
$\mathrm{G}_{\mathrm{S}}$ & $\mathrm{P}_{\max }$ & $7200 \mathrm{~kW}$ \\
& $a$ & $2,723 \mathrm{e}-6 \mathrm{~g} / \mathrm{kW}^{2}$ \\
& $b$ & $0,112 \mathrm{~g} / \mathrm{kW}$ \\
& $c$ & $120,96 \mathrm{~g}$ \\
& $\mathrm{P}_{\min }$ & $8410 \mathrm{~kW}$ \\
$\mathrm{P}_{\mathrm{B}}$ & $a$ & $16500 \mathrm{~kW}$ \\
& $b$ & $1,19 \mathrm{e}-6 \mathrm{~g} / \mathrm{kW}$ \\
& $c$ & $0,105 \mathrm{~g} / \mathrm{kW}$ \\
& & $311,85 \mathrm{~g}$ \\
\hline \hline
\end{tabular}

The cost values presented since (8) up to (14) are shown in table III.

TABLE III

PARAMETERS FOR THE CASE STUDY

\begin{tabular}{|c|c|}
\hline Parameters & Values \\
\hline $\begin{array}{c}\text { Cost } \text { Load shed } € / M W h \\
\text { Cost } \text { Wind curtailment } € / M W h \\
\text { Cost }{ }_{\text {Minimum violation } € / M W h} \\
\text { Cost } t_{\text {Fuel } \$ / g} \\
\text { Probability Contingency \% } \\
\text { Cost Blackout } € \\
\text { Cost Start-up GS } € \\
\text { Cost Start-up GB } € \\
\text { Threshold } € \\
\end{array}$ & $\begin{array}{c}1200 \\
150 \\
157,5 \\
0,0007 \\
1,5 \\
10000 \\
100 \\
150 \\
1000 \\
\end{array}$ \\
\hline
\end{tabular}

The economic impact of an energy interruption, due to load shed for instance, depends on the interruption cost $\left(€ \mathrm{MWh}^{-1}\right)$ and the amount of unserved energy (MWh). The interruption costs must be obtained from specific studies which assess the damages caused by the supply interruption to each class of consumers like, residential, commercial and industrial. It also depends on several characteristics such as duration, frequency, duration of occurrence, depth of curtailment, the existence of a warning time, and geographical coverage [20],[21]. As in this work these issues were not a subject of study, the unit interruption cost were assumed as $1200 € / \mathrm{MWh}$. The wind curtailment is an operational issue which will result on a costly operation to the system operator (because the thermal units have to cover the power curtailed). Considering the average cost of thermal production, it was considered a penalty of $150 €$ for each MWh of wind curtailed (average marginal cost of thermal units). As the thermal units have to guarantee the constancy of the frequency, the loss of the total thermal production should lead to a blackout, which must be avoided at all costs and for this the blackout cost was set to $10000 €$. The thermal units are projected to work within their production limits burning heavy fuel oil, and the efficiency is strongly dependent on the production level. When the units have to work below the minimum, they generally burn diesel. Following this and for not extrapolating the cost function to production values below the minimum, it was chosen a constant value of $157,5 €$ for each MWh. Within the technical limits the fuel cost $C_{F U E L}$ was set to $0,0007 € / g$ (maximum price in 2012). It is assumed that the units are always preheated and starting costs are equivalent to the cost of working at minimum limit during the starting time (less than 10 minutes). The probability of unexpected outages of thermal units was set to $1,5 \%$, and the threshold used in the dynamic programming was set to 10000 .

In fig. 5 the spot forecasting as well as the $98 \%$ uncertainty interval are shown. The quality of these probabilistic forecasts can be measured regarding several indicators [22],[23] which will not be addressed in this paper.

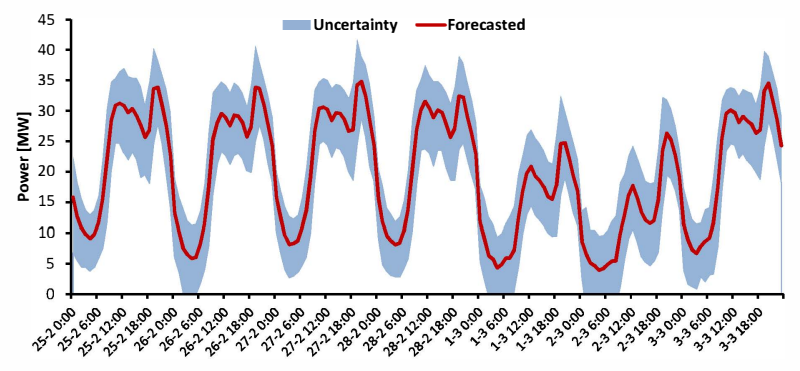

Fig. 5. Net load forecasting

Analyzing fig. 5, it should be noticed the low forecasted values during off-peak periods at $1^{\text {st }}$ and $2^{\text {nd }}$ March, with 4,3 and 3,9 MW respectively. It means that the thermal production should be very low (the minimum limit of a single $\mathrm{G}_{\mathrm{S}}$ unit is $3,84 \mathrm{MW}$ ). Analyzing the production behavior of RES, due to technical reasons, tradition is to keep the production in the geothermal $(G E O)$ power plants constant. Hydro power plants $(H)$, because of lacking storage capacity and having low rated power, do not have worth mentioning capacity of control and are strictly connected with the available resource. So, as assumed in (4), the RES with an effective and noticeable capacity of control is the wind power production with curtailment capacity. Applying this analysis to the case study, results the unit commitment showed in fig. 6 , highlighting the maximum and minimum limits of the committed GENSET. From the results depicted in fig. 6, it is visible that, except for during some off-peak periods, the GENSET limits are able to cover the spot forecasted net load necessities as well as some of the uncertainty. In the off-peak periods of February $26^{\text {th }}$ and March $1^{\text {st }}$ to $3^{\text {rd }}$ there was the possibility of keeping only 1 thermal unit online, nevertheless was preferred to curtail some wind power than increase the risk of blackout (penalized with $10000 €$, as presented in (14) ). With more than 1 unit online, the minimum limit of the GENSET increases and consequently increase the risk of wind curtailment or even operating below the limits. 


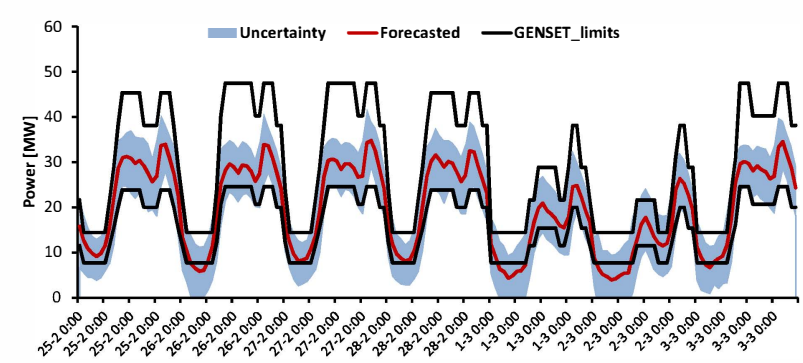

Fig. 6. Resulting scheduling

Nevertheless, due to the high penalty resulting from the $N-1$ criterion and the risk of blackout, it becomes more economical to keep on-line 2 units instead of one. On the other hand, the upper GENSET limits are generally higher than the uncertainty. This happens because of the high value of penalty associated to the probability of load shed. But even so, there were 2 hours were the GENSET's maximum production was lower than the forecasted net load. Facing the penalty for the load shed, the solution still was cheaper that the choice of other GENSET. In table IV the number of hours of load shedding, wind curtailment and the thermal units working below the minimum are shown. All these situations have associated penalty costs, shown in table III. These costs, in percentage of total risk cost are shown in table IV. As noticed above, although the reduced number of hours with load shed, the high penalty increases the contribution to the final cost.

TABLE IV

NUMBER OF HOURS OPERATING OUTSIDE THE GENSET'S LIMITS

\begin{tabular}{cccc}
\hline \hline Load & Wind & Below & Fuel \\
shedding & Curtailment & Minimum & \\
$2 \mathrm{~h}$ & $33 \mathrm{~h}$ & $4 \mathrm{~h}$ & $129 \mathrm{~h}$ \\
$2,8 \%$ & $12,9 \%$ & $0,4 \%$ & $83,8 \%$ \\
\hline \hline
\end{tabular}

\section{V.CONCLUSION}

Concerning the UC under uncertainty, it was shown that an approach based on risk assessment is a technique which allows reaching acceptable results. The problem was studied based on real environment which adds some additional complexity, meaning that there are always some parameters that are not controllable as the errors in the measures and forecasts. It should be noticed that the assessments were done based on hourly average values, which mean that intra hour phenomena may occur that were not taken into account. It was considered that the units start in the beginning of the hours and the starting time is neglected. In the classical scheduling approach, the demand is known and the algorithm tries to find a set of generation units (feasible solutions) that are able to feed the load with minor cost or with the lowest penalties in case of imbalances. In this work, the opposite paradigm was considered. Once known the available production of each GENSET, it was determined which portion of the load could be fed, based on risk assessment and associated costs. With this approach, there were no infeasible solutions. All solutions are accepted provided that the risk and consequent cost was acceptable. Hence, the trade-off between economic issues and the reliability of the system was evaluated. It was also observed that the scheduling of the thermal units in São Miguel Island is a remarkable challenge, being very hard to determine an appropriate scheduling to avoid simultaneously wind curtailment and load shed..

\section{REFERENCES}

[1] P. Fonte, B. Santos, C. Monteiro, J. Catalão, and F. P. Maciel Barbosa "Renewable Power Forecast to Scheduling of Thermal Units," in Proc. of DoCEIS'13 - Doctoral Conference on Computing, Electrical and Industrial Systems, 2014.

[2] J. Wang, A. Botterud, V. Miranda, C. Monteiro, and G. Sheble, "Impact of wind power forecasting on unit commitment and dispatch," in 8th Int. Workshop on Large-Scale Integration of Wind Power into Power Systems, Bremen, Germany, 2009, p. 8.

[3] E. Constantinescu, V. Zavala, M. Rocklin, and S. Lee, "Unit commitment with wind power generation: integrating wind forecast uncertainty and stochastic programming.," New York, 2009.

[4] J. Wang, a. Botterud, R. Bessa, H. Keko, L. Carvalho, D. Issicaba, J. Sumaili, and V. Miranda, "Wind power forecasting uncertainty and unit commitment," Appl. Energy, vol. 88, no. 11, pp. 4014-4023, Nov. 2011.

[5] D. Pozo and J. Contreras, "A Chance-Constrained Unit Commitment With an Security Criterion and Significant Wind Generation," ieeexplore.ieee.org, vol. 28, no. 3, pp. 2842-2851, 2013.

[6] G. Liu and K. Tomsovic, "Quantifying Spinning Reserve in Systems With Signi fi cant Wind Power Penetration," IEEE Trans. Power Syst., vol. 27, no. 4, pp. 2385-2393, 2012

[7] M. Shahidehpour, "Security-Constrained Unit Commitment With Volatile Wind Power Generation," IEEE Trans. Power Syst., vol. 23, no. 3, pp. 1319 1327, Aug. 2008

[8] L. Chen and Z. Y. Li, "Optimal reserve dispatch and security-constrained unit commitment considering volatile wind," 2013 IEEE Int. Conf. IEEE Reg. 10 (TENCON 2013), pp. 1-6, Oct. 2013

[9] B. Zhou and Quanyuan Jiang, "Security-Constrained Unit Commitment with Wind Power Generation by Using Interval Linear Programming," in Power and Energy Society General Meeting, 2012 IEEE, 2012, pp. 1-6.

[10] H. Wu and M. Shahidehpour, "Stochastic SCUC Solution With Variable Wind Energy Using Constrained Ordinal Optimization," Sustain. Energy, IEEE Trans., vol. 5, no. 2, pp. 379-388, 2013.

[11] Qianfan Wang; Yongpei Guan; Jianhui Wang, "A Chance-Constrained TwoStage Stochastic Program for Unit Commitment With Uncertain Wind Power Output," Power Syst. IEEE Trans., vol. 27, no. 1, pp. 206-215, 2012.

[12] L. Wu, M. Shahidehpour, and Z. Li, "Comparison of Scenario-Based and Interval Optimization Approaches to Stochastic SCUC," IEEE Trans. Power Syst., vol. 27, no. 2, pp. 913-921, 2012.

[13] M. E. Khodayar, M. Shahidehpour, L. Wu, and A. Variables, "Enhancing the Dispatchability of Variable Wind Generation by Coordination With PumpedStorage Hydro Units in Stochastic Power Systems," vol. 28, no. 3, pp. 2808 2818, 2013.

[14] H. Chen, H. Li, R. Ye, and B. Luo, "Robust scheduling of power system with significant wind power penetration," in Power and Energy Society General Meeting, 2012 IEEE, 2012, pp. 1-5.

[15] A. Kalantari, J. F. Restrepo, and F. D. Galiana, "Security-Constrained Unit Commitment With Uncertain Wind Generation: The Loadability Set Approach," vol. 28, no. 2, pp. 1787-1796, 2013.

[16] M. Matos and R. J. Bessa, "Setting the Operating Reserve Using Probabilistic Wind Power Forecasts," IEEE Trans. Power Syst., vol. 26, no. 2, pp. 594 603, May 2011.

[17] N. Di Domenica, G. Mitra, P. Valente, and G. Birbilis, "Stochastic programming and scenario generation within a simulation framework: An information systems perspective," Decis. Support Syst., vol. 42, no. 4, pp. 2197-2218, Jan. 2007.

[18] V. Botterud, A.; Zhou, Z.; Wang, J.; Valenzuela, J.; Sumaili, J.; Bessa, R.J.; Keko, H.; Miranda, "Unit commitment and operating reserves with probabilistic wind power forecasts," in PowerTech, 2011 IEEE Trondheim, 2011, pp. 1-7.

[19] X. Xia and a. M. Elaiw, "Optimal dynamic economic dispatch of generation A review," Electr. Power Syst. Res., vol. 80, no. 8, pp. 975-986, Aug. 2010.

[20] A. M. L. da Silva, G. Perez A., J. W. Marangon Lima, and J. C. O. Mello, "Loss of load costs in generating capacity reliability evaluation," Electr. Power Syst. Res., vol. 41, no. 2, pp. 109-116, May 1997.

[21] L. Manso, A. Silva, and J. Mello, "Comparison of alternative methods for evaluating loss of load costs in generation and transmission systems," Electr. Power Syst. Res., vol. 50, pp. 107-114, 1999.

[22] R. Bessa, J. Sumaili, V. Miranda, A. Botterud, J. Wang, and E. Constantinescu, "Time-adaptive kernel density forecast: A new method for wind power uncertainty modeling," in 17th Power System Computation Conference, 2010. 